\title{
Erratum: Light probes in a strongly coupled anisotropic plasma [Phys. Rev. D 87, 046004 (2013)]
}

\author{
Berndt Müller and Di-Lun Yang
}

(Received 15 September 2018; published 25 September 2018)

DOI: 10.1103/PhysRevD.98.069903

\section{STOPPING DISTANCES AT SMALL ANISOTROPY}

The second line of Eq. (5) should be

$$
\mathcal{B}_{2}(z)=-\frac{z_{h}^{2}}{24}\left[\frac{10 z^{2}}{z_{h}^{2}+z^{2}}+\log \left(1+\frac{z^{2}}{z_{h}^{2}}\right)\right] .
$$

A minor typo of $\mathcal{B}_{2}(z)$ is corrected here.

There were errors in the numerical code for computing the ratios of stopping distances by varying anisotropy with fixed temperature, entropy density, and energy density, respectively. The correct results are shown in Figs. 1-3 below. The qualitative features of $R_{x}$ along the longitudinal direction remain unchanged in all the cases. However, for $R_{x}$ along the transverse direction, the decrease by increasing anisotropy becomes milder when fixing temperature or energy density. For the case with fixed entropy density, $R_{x}$ along the transverse direction even increases with anisotropy. Nonetheless, the

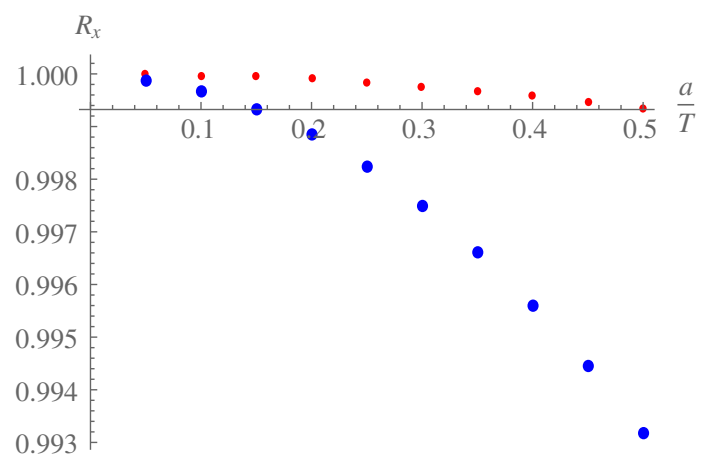

FIG. 1. $R_{x}=x_{\text {aniso }} / x_{\text {iso }}$ represents the ratio of the stopping distances in the Mateos and Trancanelli (MT) geometry with anisotropy to without anisotropy, where $x_{\text {aniso }}=x_{T}$ for the red points and $x_{\text {aniso }}=x_{L}$ for the blue points. Here we take $|\vec{q}|=0.99 \omega$ and fix the temperature of media.

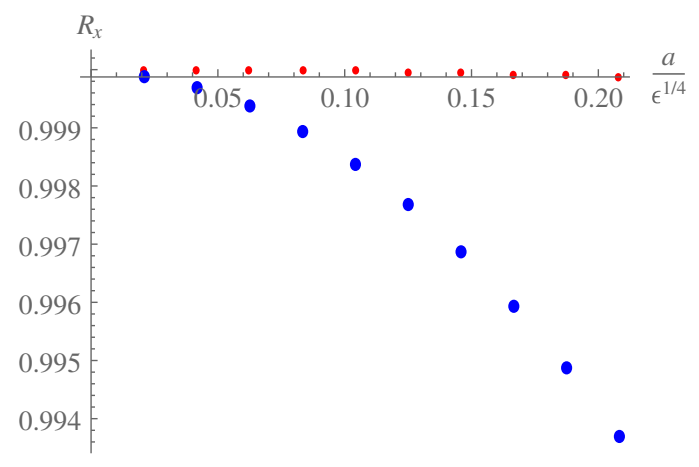

FIG. 2. $R_{x}=x_{\text {aniso }} / x_{\text {iso }}$ represents the ratio of the stopping distances in the MT geometry with anisotropy to without anisotropy, where $x_{\text {aniso }}=x_{T}$ for the red points and $x_{\text {aniso }}=x_{L}$ for the blue points. Here we take $N_{c}=3,|\vec{q}|=0.99 \omega$ and fix the energy density of media. 


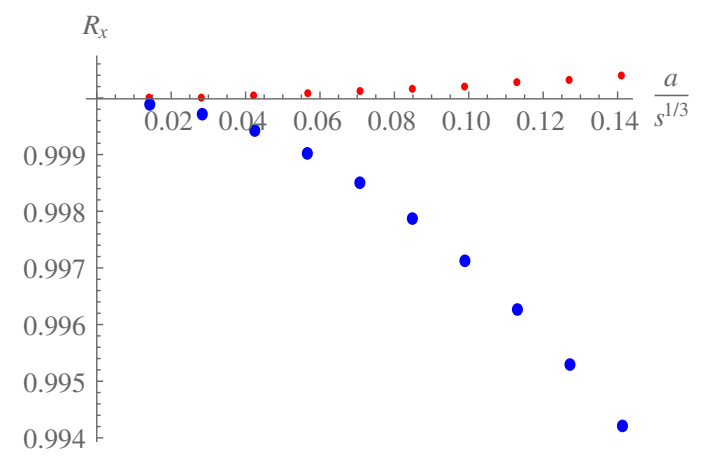

FIG. 3. $R_{x}=x_{\text {aniso }} / x_{\text {iso }}$ represents the ratio of the stopping distances in the MT geometry with anisotropy to without anisotropy, where $x_{\text {aniso }}=x_{T}$ for the red points and $x_{\text {aniso }}=x_{L}$ for the blue points. Here we take $N_{c}=3,|\vec{q}|=0.99 \omega$ and fix the entropy density of media.

correct results at small anisotropy are more consistent with the full numerical calculations at mid and large anisotropy. In summary, the conclusion in the original paper remains unchanged.

The authors thank Raguram Subramaniam for pointing out the mistakes. 